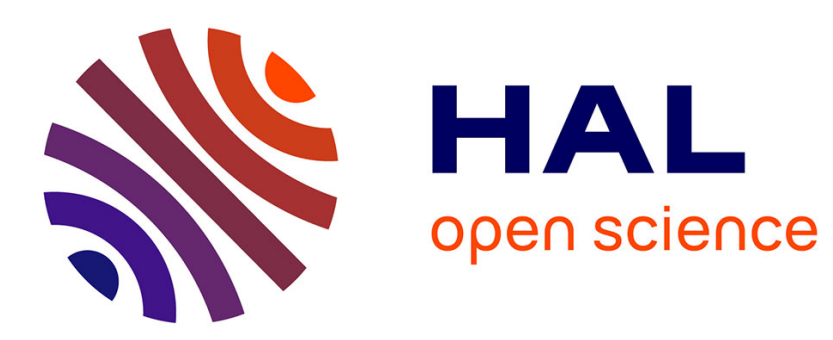

\title{
Ordre atomique et spectrométrie Mössbauer dans l'alliage Fe-Co équiatomique
}

\author{
J.P. Eymery, P. Moine
}

\section{To cite this version:}

J.P. Eymery, P. Moine. Ordre atomique et spectrométrie Mössbauer dans l'alliage Fe-Co équiatomique. Journal de Physique Lettres, 1978, 39 (2), pp.23-28. 10.1051/jphyslet:0197800390202300 . jpa00231434

\section{HAL Id: jpa-00231434 https://hal.science/jpa-00231434}

Submitted on 1 Jan 1978

HAL is a multi-disciplinary open access archive for the deposit and dissemination of scientific research documents, whether they are published or not. The documents may come from teaching and research institutions in France or abroad, or from public or private research centers.
L'archive ouverte pluridisciplinaire HAL, est destinée au dépôt et à la diffusion de documents scientifiques de niveau recherche, publiés ou non, émanant des établissements d'enseignement et de recherche français ou étrangers, des laboratoires publics ou privés. 


\title{
ORDRE ATOMIQUE ET SPECTROMÉTRIE MÖSSBAUER DANS L'ALLIAGE Fe-Co ÉQUIATOMIQUE
}

\author{
J. P. EYMERY et P. MOINE
}

Laboratoire de Métallurgie Physique $(*)$, 40, Avenue du Recteur-Pineau, 86022 Poitiers, France

(Reçu le 26 septembre 1977, accepté le 5 décembre 1977)

\begin{abstract}
Résumé. - On étudie les variations du champ interne et du déplacement isomérique dans l'alliage $\mathrm{Fe}$-Co (50-50) en fonction de l'ordre atomique et de la manière dont on fait varier ce dernier : trempe depuis des températures inférieures à $T_{\mathrm{c}}$ et restauration de l'ordre à partir d'un état initial désordonné. On discute également la nature de la transformation désordre-ordre.
\end{abstract}

\begin{abstract}
The variation in both hyperfine field and isomer shift are studied in equiatomic $\mathrm{Fe}-\mathrm{Co}$ alloy as a function of atomic order. Two kinds of experiments are carried out : quenching from below $T_{\mathrm{c}}$ and order restoration in initially disordered material. The nature of the disorder to order transformation is also discussed.
\end{abstract}

1. Introduction. - L'étude par effet Mössbauer des phénomènes d'ordre atomique s'est développée ces dernières années. Les composés ordonnés à longue distance qui ont le plus fait l'objet d'investigations sont les fer-aluminium, fer-nickel et fersilicium [1]. L'ordre à courte distance a également été étudié; un article de Schwartz et Asano [2] fait une analyse critique des publications antérieures à 1974 relatives à la mesure des paramètres d'ordre à courte distance de Warren à partir de spectres Mössbauer d'alliages de fer ferromagnétiques.

Dans le cas de l'alliage Fe-Co équiatomique, seuls les états d'ordre extrêmes ont été analysés $[3,4,5,6]$. Cette note est relative au même composé; elle a pour but de déterminer les variations des paramètres Mössbauer en fonction de l'état d'ordre à longue distance. L'alliage $\mathrm{Fe}-\mathrm{Co}(50-50)$ se prête bien à une telle étude puisque, dans son cas, il est possible de faire varier le degré d'ordre à longue distance de deux façons différentes. Une première méthode consiste à effectuer des trempes à l'eau dans la gamme de température $600-900{ }^{\circ} \mathrm{C}$. Une deuxième méthode consiste à tremper les échantillons au-dessus de la température critique de la transition ordre-désordre $\left(T_{\mathrm{c}} \# 730^{\circ} \mathrm{C}\right)$ et à restaurer l'ordre par revenu entre 400 et $500^{\circ} \mathrm{C}$. Les deux processus seront étudiés successivement; il sera en particulier mis l'accent sur la nature de la transformation ordre-désordre. L'ordre à longue distance est décrit par le paramètre $\eta=2 p-1$ où $p$ désigne la fraction d'atomes $\mathrm{A}$ sur les' sites $\alpha$ et également la fraction d'atomes B sur les sites $\beta$.

(*) L.A. 131 du C.N.R.S.
2. Techniques expérimentales. - $\mathrm{La}$ coulée de Fe-Co utilisée a la composition pondérale suivante : $50,1 \% \mathrm{Fe}$ et $49,9 \%$; elle comporte en outre quelques p.p.m de $\mathrm{C}, \mathrm{Si}, \mathrm{S}, \mathrm{P}$ et $\mathrm{Mn}$. La forme initiale des échantillons est un disque de $12 \mathrm{~mm}$ de diamètre et de $0,5 \mathrm{~mm}$ d'épaisseur.

Les éprouvettes subissent tout d'abord un recuit d'une heure à $850{ }^{\circ} \mathrm{C}$ suivi d'un refroidissement jusqu'à l'ambiante à la vitesse de $50{ }^{\circ} \mathrm{C} / \mathrm{h}$; ce traitement conduit à l'état d'ordre maximum caractérisé par une taille des domaines ordonnés voisine de $3700 \AA$ et un paramètre d'ordre à longue distance $\eta_{\mathrm{M}} \# \mathbf{0 , 9 8 \text { . }}$

L'étude des états d'ordre après trempe à l'eau [7] montre que la taille des domaines d'ordre ne dépend pas de la température de trempe (en dessous de $T_{\mathrm{c}}$ ) et reste sensiblement égale à celle observée après recuit; seul le degré d'ordre décroît de $\eta_{M}$ vers 0 entre l'ambiante et $T_{\mathrm{c}}$. Les trempes sont effectuées par chute de l'échantillon dans l'eau après maintien de $30 \mathrm{~min}$. à la température désirée.

Après les traitements thermiques, les échantillons sont amincis jusqu'à $25 \mu \mathrm{m}$ tout d'abord par abrasion mécanique puis par polissage électrolytique dans un bain comprenant $10 \%$ de butycellosolve, $12 \%$ d'eau, $8 \%$ d'acide perchlorique et $70 \%$ d'alcool éthylique.

Le spectromètre Mössbauer utilisé est le modèle A.M.E. $40 \mathrm{C}$ d'Elscint. Il fonctionne avec une source de ${ }^{57} \mathrm{Co}$ dispersé dans du rhodium $(25 \mathrm{mCi})$, un analyseur à 1024 canaux et un miniordinateur Elbit 100. Les spectres sont réalisés en accélération constante à la température ordinaire. Les enregistrements exploités correspondent à un nombre de coups 
supérieur ou égal à $1,5 \times 10^{6}$ au niveau du fond continu. Tous les dépouillements sont effectués en prenant le fer $\alpha$ comme référence.

3. Résultats des essais après trempe. - 3.1 SPECTRES MösSBAUER. - Les spectres présentent six raies élargies de manière dissymétrique quel que soit le degré d'ordre. Ce phénomène, peu important il est vrai, n'avait pas été signalé dans les publications précédentes $[4,5,6]$. L'absence de satellites apparents permet de faire un ajustement à un sextuplet de lorentziennes. Le programme de calcul numérique réalisé à cet effet utilise une méthode des moindres carrés; sa technique est inspirée de celle publiée par plusieurs auteurs notamment Rhodes et al. [8] et Le Caer [9].

La dissymétrie des spectres peut être caractérisée par le rapport $\Gamma_{1} / \Gamma_{6}$ où $\Gamma_{1}$ et $\Gamma_{6}$ désignent les largeurs à mi-hauteur des raies extrêmes, les indices croissant comme les vitesses; expérimentalement on trouve $\Gamma_{1} / \Gamma_{6}$ compris entre 1,10 et 1,15 pour tous les états d'ordre $\eta<\eta_{M}$; pour $\eta_{M}$ la valeur du rapport est légèrement inférieure, voisine de 1,06. Dans ce dernier cas, le faible écart à l'unité provient sans doute du fait que $\eta_{\mathrm{M}}$ est légèrement inférieur à 1 ainsi que de l'incertitude sur la mesure de $\Gamma_{1}$ et $\Gamma_{6}$.

La figure $1 a$ montre les variations de $\frac{1}{2}\left(\Gamma_{1}+\Gamma_{6}\right)$ en fonction de la température de trempe $T_{\mathrm{t}}$. Entre
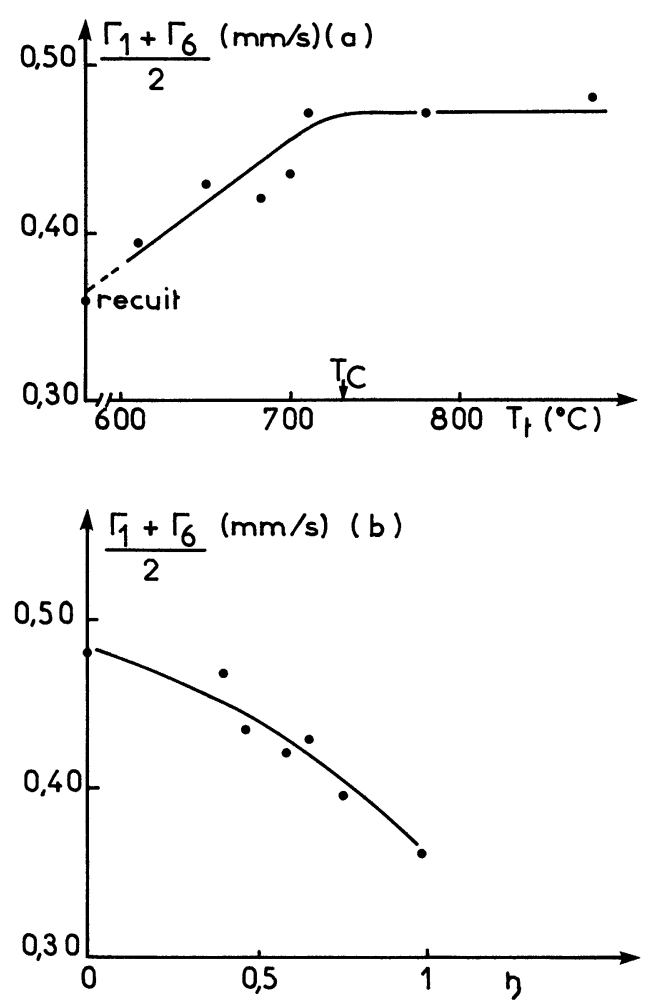

Fig. 1. - Variations de $\frac{\Gamma_{1}+\Gamma_{6}}{2}$ en fonction de la température de trempe $T_{\mathrm{t}}$.

$\left[\right.$ Variations in $\frac{\Gamma_{1}+\Gamma_{6}}{2}$ as a function of quenching temperature $\left.T_{v}.\right]$ l'état recuit $\left(\eta_{\mathrm{M}}\right)$ et $T_{\mathrm{c}}$, la largeur à mi-hauteur croît de $0,36 \mathrm{~mm} / \mathrm{s} \mathrm{à} 0,48 \mathrm{~mm} / \mathrm{s}$; au-dessus de $T_{\mathrm{c}}$ et jusqu'à la température de $880^{\circ} \mathrm{C}$, la largeur des raies extrèmes garde une valeur sensiblement constante. Sur la figure $1 b$ on a porté les variations de $\frac{1}{2}\left(\Gamma_{1}+\Gamma_{6}\right)$ en fonction du degré d'ordre à longue distance $\eta$ mesuré par diffraction des rayons X [7]; on constate alors une décroissance monotone de la largeur des raies en fonction du degré d'ordre.

L'effet quadrupolaire moyen est négligeable pour tous les états d'ordre; en effet expérimentalement on trouve des valeurs toujours inférieures à $0,003 \mathrm{~mm} / \mathrm{s}$ et sans rapport avec l'ordre.

\subsection{VARIATIONS DU CHAMP INTERNE EN FONCTION} DU DEGRÉ D'ORDRE. - Le dépouillement des spectres conduit à une valeur moyenne du champ interne notée $H^{*}$; dans la suite nous ne considérerons que la valeur absolue du champ. Les variations de $H^{*}$ en fonction de la température de trempe et du degré d'ordre sont reportées sur les figures $2 a$ et $2 b$. Entre les états d'ordre extrêmes, $H^{*}$ varie de 341,5 à $350,5 \mathrm{kOe}$ ce qui constitue un bon accord avec les résultats de de Mayo et al. [4] et de Vincze et al. [5].

Dans le cas des alliages ordonnés et en particulier de $\mathrm{Ni}_{3} \mathrm{Fe}$, l'application de la formule semi-empirique :

$$
H\left(m_{1}, m_{2}\right)=H_{0}+m_{1} \Delta H_{1}+m_{2} \Delta H_{2}
$$
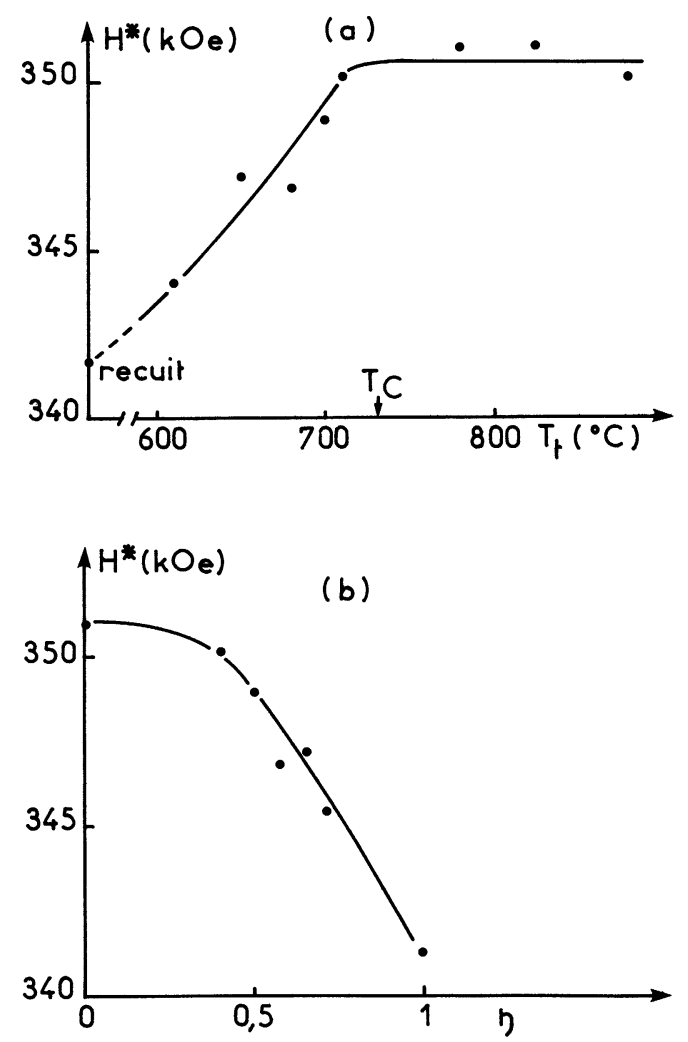

FIG. 2. - Variations du champ interne en fonction (a) de la température de trempe et $(b)$ du degré d'ordre à longue distance $\eta$.

[Variation in hyperfine field as a function of $(a)$ the quenching temperature and $(b)$ the long range order parameter $\eta$.] 
s'est avérée fructueuse pour décrire les variations du champ interne en fonction de l'ordre atomique [10] ; dans cette relation $m_{1}$ et $m_{2}$ sont les nombres d'atomes de fer respectivement premiers et seconds voisins, de plus le terme $H_{0}$ est supposé constant. Il nous a paru délicat d'appliquer cette formule au cas du Fe-Co car l'aimantation à saturation de ce composé varie avec l'état d'ordre [11] ce qui empêche de supposer $H_{0}$ constant.

Dans un premier temps nous avons analysé les variations de $H^{*}$ en fonction de l'ordre atomique par rapport aux variations du moment magnétique de l'atome de fer et du moment moyen par atome. En effet le champ interne pour un atome de fer entouré de $n_{1}$ atomes de cobalt premiers voisins peut se mettre sous la forme :

$$
H\left(n_{1}\right)=a \mu_{\mathrm{Fe}}\left(n_{1}\right)+b \bar{\mu}
$$

où $\mu_{\mathrm{Fe}}\left(n_{1}\right)$ est le moment magnétique de l'atome de fer entouré de $n_{1}$ atomes de cobalt premiers voisins et $\bar{\mu}$ le moment moyen par atome de l'alliage; $a$ et $b$ sont des constantes dont les valeurs pour l'alliage $\mathrm{Fe}-\mathrm{Co}$ équiatomique désordonné sont d'après Vincze et al. [5] :

$$
a=70 \mathrm{kOe} / \mu_{\mathrm{B}} \quad b=63,5 \mathrm{kOe} / \mu_{\mathrm{B}} .
$$

D'après Kouvel [11] et Collins et Forsyth [12], le moment magnétique du cobalt $\mu_{\mathrm{C}_{0}}$ est indépendant de l'entourage et égal à $1,8 \mu_{\mathrm{B}}$, seul $\mu_{\mathrm{Fe}}$ varie en fonction de $n_{1}$. Dans ces conditions $\bar{\mu}$ peut être calculé par la relation :

$$
\bar{\mu}=0,5\left[\mu_{\mathrm{Co}}+\sum_{n_{1}} \mu_{\mathrm{Fe}}\left(n_{1}\right) W\left(n_{1}\right)\right]
$$

où $W\left(n_{1}\right)$ est la probabilité pour qu'un atome de fer soit entouré de $n_{1}$ atomes de cobalt premiers voisins. Le champ moyen $\bar{H}$ se déduit alors de $H\left(n_{1}\right)$ par la formule :

$$
\bar{H}=\sum_{n_{1}} W\left(n_{1}\right) H\left(n_{1}\right)
$$

La combinaison des formules (1), (2) et (3) conduit à :

$$
\bar{H}=a\left(2 \bar{\mu}-\mu_{\mathrm{Co}}\right)+b \bar{\mu} .
$$

La comparaison des valeurs expérimentales $H^{*}$ aux valeurs moyennes théoriques $\bar{H}$ nécessite le choix d'une distribution des atomes de cobalt premiers voisins permettant de calculer $W\left(n_{1}\right)$.

Schwartz et Asano [2] ont critiqué le processus, fréquemment rencontré dans la littérature, qui consiste à calculer $W\left(n_{1}\right)$ à partir des coefficients de Cowley en supposant une distribution binomiale des $n_{1}$. Drijver [10] a proposé un autre mode de calcul des $W\left(n_{1}\right)$ valable dans le cas de l'ordre à longue distance ; $W\left(n_{1}\right)$ est déduit des probabilités d'occupation des sites $\alpha$ et $\beta$ et par suite du paramètre d'ordre à longue distance; le processus ne suppose une répartition au hasard que pour les atomes mal placés. Son application au cas du type B2 stœchiométrique conduit à :

$$
W\left(n_{1}\right)=p P_{8}\left(p ; n_{1}\right)+(1-p) P_{8}\left(1-p ; n_{1}\right)
$$

où $P_{k}(p ; j)$ est la distribution binomiale définie par :

$$
P_{k}(p, j)=\frac{k !}{j !(k-j) !} p^{j}(1-p)^{k-j}
$$

Les valeurs de $W\left(n_{1}\right)$ en fonction de $n_{1}$ sont reportées sur les diagrammes de la figure 3 pour diverses valeurs de $\eta$. La figure 4 donne alors les variations de $\bar{\mu}$ en fonction de $\eta$ d'après la relation (2); les valeurs de $\mu_{\mathrm{Fe}}\left(n_{1}\right)$ nécessaires aux calculs ont été tirées des références [11] et [12]. On peut constater que $\bar{\mu}$ croît de 2,40 à $2,475 \mu_{\mathrm{B}}$ entre $\eta=0$ et $\eta=1$ alors que le champ interne $H^{*}$ décrôit avec l'ordre. L'application de la formule (4) avec $a=70 \mathrm{kOe} / \mu_{\mathrm{B}}$ conduit alors à $b$ variable avec l'état d'ordre comme le montre la figure 5. Pour l'ordre minimum on trouve $b=59 \mathrm{kOe} / \mu_{\mathrm{B}}$ ce qui constitue un bon accord avec la valeur de $63,5 \mathrm{kOe} / \mu_{\mathrm{B}}$ proposée par Vincze et al. [5]; à l'état d'ordre maximum on trouve $b=49 \mathrm{kOe} / \mu_{\mathrm{B}}$. Vincze et al. [5] ont montré que $b$
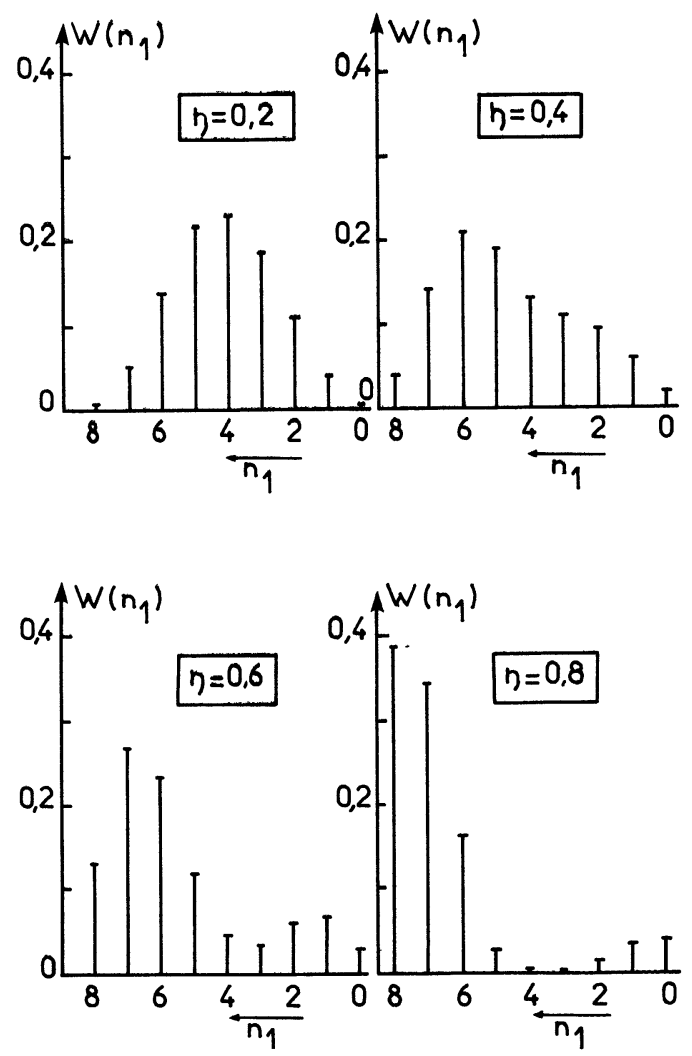

FIG. 3. - Probabilités de trouver $n_{1}$ atomes de cobalt en premiers voisins autour d'un atome de fer en fonction du degré d'ordre à longue distance.

[Probability of having $n_{1}$ cobalt atoms around a central iron atom in the first neighbouring shell as a function of the long range order parameter.] 


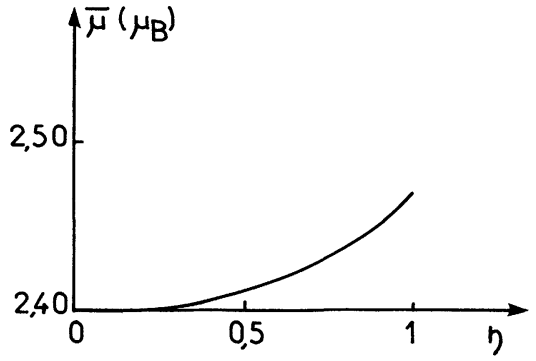

Fig. 4. - Variations du moment magnétique moyen par atome $\bar{\mu}$ en fonction du degré d'ordre à longue distance.

[Variation in average moment per atom as a function of the long range order parameter.]

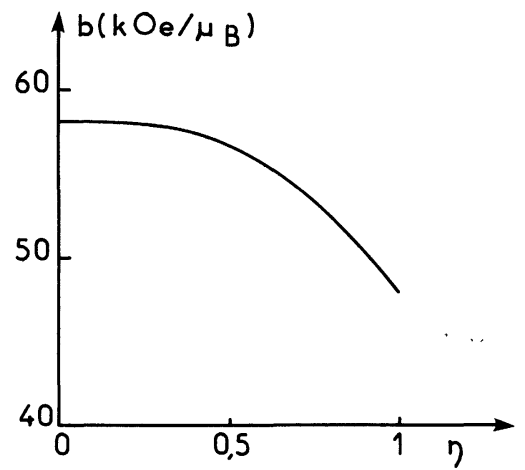

Fig. 5. - Variations du terme $b$ du champ interne en fonction du degré d'ordre à longue distance.

[Variation in the $b$ term of the hyperfine field as a function of the long range order parameter.]

variait avec la concentration en cobalt dans les alliages fer-cobalt désordonnés; nos calculs indiquent qu'à concentration constante, il faut admettre que $b$ varie avec l'état d'ordre. Cet ensemble de résultats montre que la formule (1) ne reflète que très approximativement le comportement des alliages Fe-Co.

Une autre analyse simplifiée inspirée de l'article de Muraoka et al. [13] consiste à écrire que le champ au noyau de fer $H_{\mathrm{Fe}}$ peut se mettre sous la forme :

$$
H_{\mathrm{Fe}}=a \mu_{\mathrm{Fe}}+b_{1} \sum_{n} \mu_{1 n}
$$

où $\mu_{1 n}$ est le moment magnétique d'un atome premier voisin d'un atome de fer. L'application de la formule (1 bis) pour l'état d'ordre maximum conduit à $b_{1}=8,4 \mathrm{kOe} / \mu_{\mathrm{B}}$ pour la couche de premiers voisins en prenant les valeurs numériques suivantes : $a=70 \mathrm{kOe} / \mu_{\mathrm{B}}, \quad \mu_{\mathrm{Co}}=1,8 \mu_{\mathrm{B}}$ ainsi que

$$
H_{\mathrm{Fe}}=341,5 \mathrm{kOe}
$$

pour $\eta=\eta_{\mathrm{M}}$ et $\mu_{\mathrm{Fe}}=3,15 \mu_{\mathrm{B}}$ (cette dernière valeur étant tirée de la référence [11]). Le report de la valeur de $b_{1}$ ainsi déterminée dans la formule ( 1 bis) appliquée à l'état d'ordre minimum permet ensuite de calculer $\mu_{\mathrm{Fe}}=2,8 \mu_{\mathrm{B}}$ pour $\eta=0$ ce qui constitue un bon accord avec les résultats théoriques et expérimentaux de l'article [11]. La relation (1 bis) permet donc d'expliquer mieux que la relation (1) le sens des variations du champ interne avec l'état d'ordre.

3.3 VARIATIONS DU DÉPLACEMENT ISOMÉRIQUE EN FONCTION DE L'ORDRE. - Comme dans le cas du champ interne, le dépouillement des spectres conduit à une valeur moyenne du déplacement isomérique notée $I S^{*}$. Les variations de $I S^{*}$ en fonction de la température de trempe sont reportées sur la figure $6 a$. Entre les états extrêmes, IS* varie de 0,017 à $0,032 \mathrm{~mm} / \mathrm{s}$; au-dessus de $T_{\mathrm{c}}, I S^{*}$ reste sensiblement constant. Les valeurs de $I S^{*}$ pour les états d'ordre extrême sont en bon accord avec celles de de Mayo et al. [4] et de Vincze et al. [5].

Le déplacement isomérique $I S$ peut être représenté par la formule :

$$
I S=I S_{0}+m_{1} \Delta I S_{1}
$$

lorsqu'on se limite aux premiers voisins comme il est fait habituellement dans les alliages ordonnés [10] ; $m_{1}$ désigne le nombre d'atomes de fer en position de premiers voisins; $\Delta I S_{1}$ est la variation de déplacement isomérique due au remplacement d'un atome de cobalt par un atome de fer, enfin $I S_{0}$ représente la valeur du déplacement isomérique à l'état d'ordre maximum $\left(m_{1}=0\right)$.
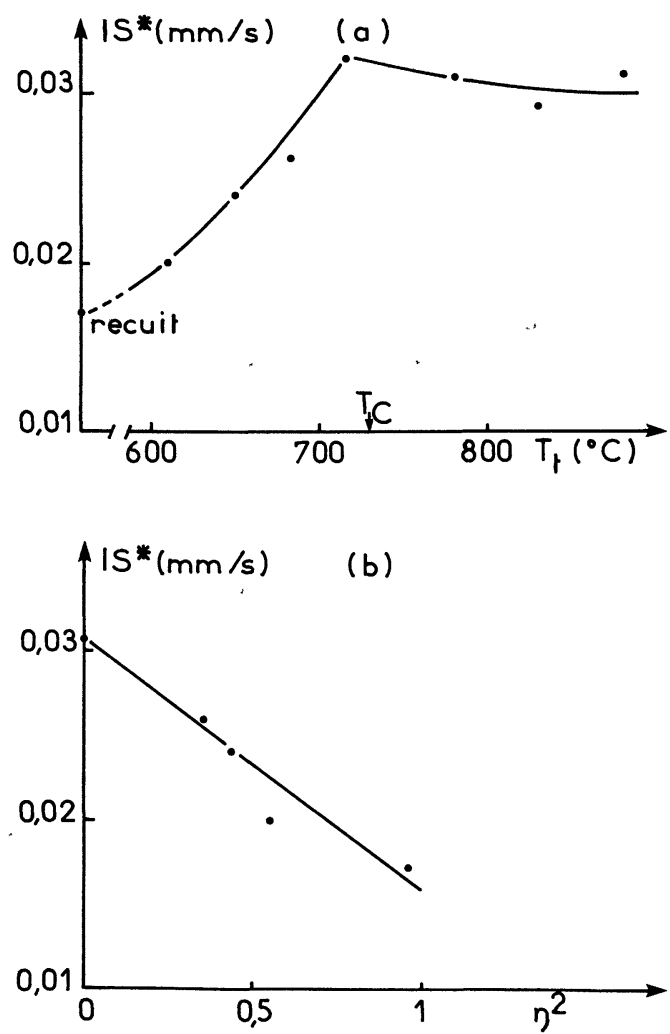

Fig. 6. - Variations du déplacement isomérique en fonction (a) de la température de trempe et $(b)$ du carré du degré d'ordre à longue distance.

[Variation in isomer shift as a function of $(a)$ the quenching temperature and $(b)$ the square of the long range order parameter.] 
Pour comparer les valeurs expérimentales $I S^{*}$ aux valeurs moyennes théoriques $\overline{I S}=I S_{0}+\bar{m}_{1} \Delta I S_{1}$ on utilise les relations suivantes :

$$
\begin{gathered}
\bar{m}_{1}=\sum_{m_{1}} m_{1} W\left(m_{1}\right) \\
W\left(m_{1}\right)=p P_{8}\left(1-p ; m_{1}\right)+(1-p) P_{8}\left(p ; m_{1}\right)
\end{gathered}
$$

où $W\left(m_{1}\right)$ est la probabilité de trouver $m_{1}$ atomes de fer en position de premiers voisins.

L'expression $\overline{I S}$ doit être linéaire en fonction de $\eta^{2}$; en effet $m_{1}$ s'exprime aussi linéairement en fonction du coefficient de Cowley $\alpha_{1}$ lui-même égal à $-\eta^{2}$. Le tracé de $I S^{*}$ en fonction de $\eta^{2}$ conduit bien à une droite comme le montre la figure $6 b$. Le calcul de $m_{1}$ à l'aide des formules (6) et (7) et l'assimilation de $\overline{I S}$ à $I S^{*}$ permettent de proposer une valeur approchée de $\Delta S I_{1}$; on trouve :

$$
\Delta I S_{1}=0,004 \mathrm{~mm} / \mathrm{s} .
$$

4. Etude de la transformation désordre-ordre. - Les cinétiques de mise en ordre ont été le plus souvent étudiées par diffraction des rayons $\mathrm{X}$, technique qui ne permet pas de déceler les états d'ordre faibles sur l'alliage Fe-Co. Il était donc intéressant d'utiliser la spectrométrie Mössbauer pour étudier en particulier le début de la mise en ordre qui renseigne sur la nature de la transformation désordre-ordre.

Une cinétique a été réalisée à la température de $480{ }^{\circ} \mathrm{C}$ sur des échantillons initialement trempés à $780^{\circ} \mathrm{C}$. Les variations de $H^{*}, I S^{*}$ et $\frac{1}{2}\left(\Gamma_{1}+\Gamma_{6}\right)$ sont portées sur la figure 7 en fonction du logarithme du temps. On peut noter une décroissance

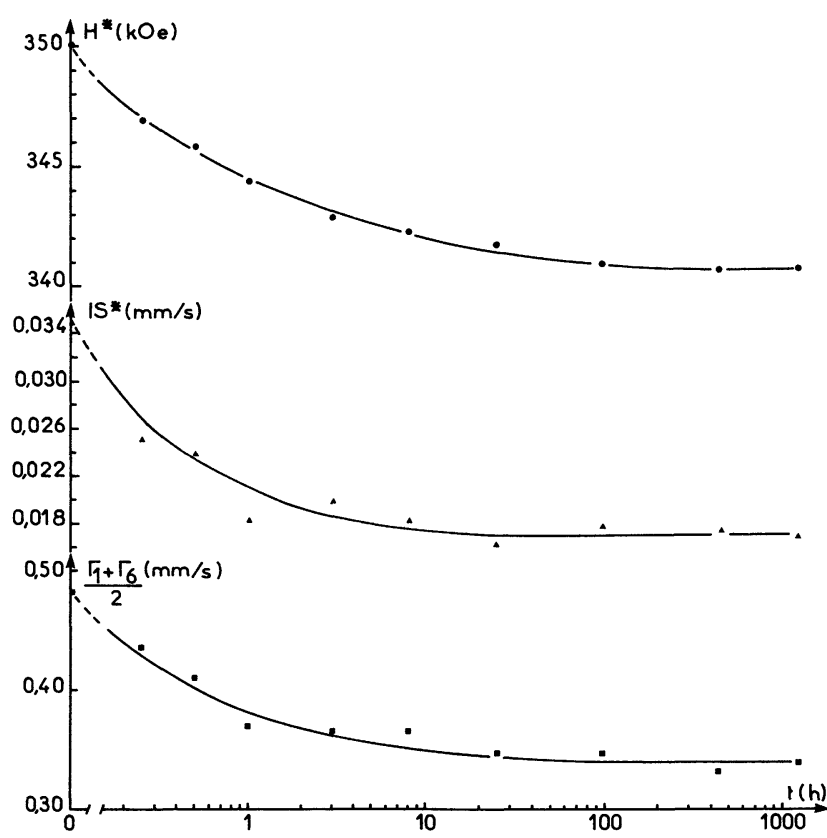

Fig. 7. - Cinétique de mise en ordre à $480{ }^{\circ} \mathrm{C}$ après trempe à $780^{\circ} \mathrm{C}$.

[Ordering kinetic at $480^{\circ} \mathrm{C}$ after quenching from $780{ }^{\circ} \mathrm{C}$.] régulière de ces paramètres au cours de la mise en ordre; leurs valeurs initiales et finales sont en accord avec les résultats du paragraphe 3 .

Pour discuter la nature de la transformation désordre-ordre, nous avons également simulé des spectres Mössbauer dans l'hypothèse d'une transformation hétérogène c'est-à-dire se produisant par germination et croissance de domaines ordonnés dans la matrice désordonnée. Les données numériques nécessaires à ces simulations ont été empruntées au paragraphe 3 ; les calculs ont été effectués en supposant le facteur de Debye-Waller indépendant de l'état d'ordre et en négligeant les effets d'épaisseur et de polarisation. Les largeurs $\Gamma$ des pics extrêmes du spectre présentent alors un accroissement en début de cinétique comme le montre la figure 8 qui donne les variations du rapport $\Gamma / \Gamma_{\mathrm{DES}}$. en fonction de la fraction de volume ordonné $X\left(\Gamma_{\text {Des. }}\right.$ désigne la valeur de $\Gamma$ pour l'état désordonné initial); chaque courbe de la figure 8 est indexée par une valeur de $\eta$ qui est le paramètre d'ordre à l'intérieur des zones ordonnées supposé constant au cours de la cinétique.

Les surstructures de type $\mathrm{L} 2{ }_{0}$ ont longtemps fourni l'exemple classique des transitions du second ordre thermodynamique. En fait la découverte de domaines ordonnés dans $\mathrm{Fe}-\mathrm{Co}$ et $\mathrm{Fe}-\mathrm{Co}-2 \mathrm{~V}$ [14, $15,16]$ était un argument suffisant pour remettre en cause l'existence d'un processus homogène. Dans une étude récente [17] effectuée par diffraction des rayons $\mathrm{X}$, nous avons montré que le premier stade de la transformation désordre-ordre n'obéissait pas aux règles classiques de la germination-croissance et pouvait être interprété comme une mise en ordre homogène. Les résultats de spectrométrie Mössbauer apportent un argument supplémentaire au rejet du processus de germination-croissance. En effet la simulation des spectres a montré qu'un tel processus devrait conduire à un élargissement notable des

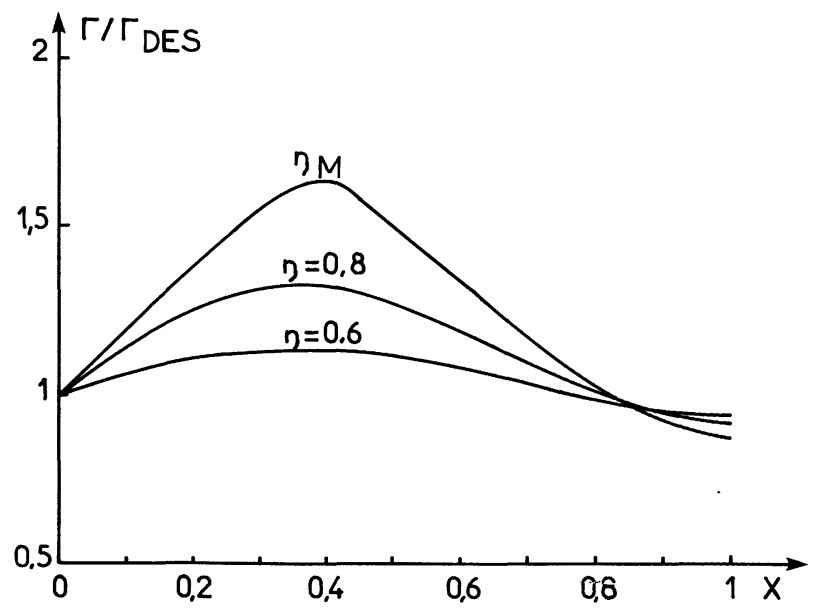

FIG. 8. - Variations du rapport $\Gamma / \Gamma_{\text {DEs. }}$ en fonction de la fraction de volume ordonné $X$.

[Variation in the ratio $\Gamma / \Gamma_{\mathrm{DES}}$ as a function of the ordered volume fraction $X$.] 
pics extrêmes alors qu'expérimentalement on observe (Fig. 7) une décroissance monotone dès le début de la cinétique.

5. Conclusion. - Le champ interne et le déplacement isomérique ont été étudiés en fonction de l'ordre atomique dans l'alliage $\mathrm{Fe}-\mathrm{Co}(50-50)$. Il s'est avéré que la formule : $H\left(n_{1}\right)=a \mu_{\mathrm{Fe}}\left(n_{1}\right)+b \bar{\mu}$ ne donnait pas une description satisfaisante du champ interne, les résultats expérimentaux conduisant à supposer $b$ variable avec l'état d'ordre. Par contre la relation
$H_{\mathrm{Fe}}=a \mu_{\mathrm{Fe}}+b_{1} \sum_{n} \mu_{1 n}$.reflète mieux le sens des variations du champ avec l'ordre atomique. Les variations du déplacement isomérique peuvent être analysées à l'aide de la formule : IS $=I S_{0}+m_{1} \Delta I S_{1}$; les dépouillements ont conduit à proposer :

$$
\Delta I S_{1}=0,004 \mathrm{~mm} / \mathrm{s} \text {. }
$$

Enfin l'étude d'une cinétique de mise en ordre a fourni des arguments pour rejeter une transformation se produisant par germination-croissance.

\section{Bibliographie}

[1] Applications of Mössbauer Spectroscopy, edited by R. L. Cohen · [9] LE CAER, G., Thèse de Doctorat, Nancy (1974). 1976 vol. 1 p. 66.

[2] Schwartz, L. H, et Asano, A., J. Physique C 6 (1974) C6-453.

[3] Johnson, C. E., Ridout, M. S. et Cranshaw, T. E., Proc. Phys. Soc. 81 (1963) 1079.

[4] De Mayo, B., Forester, D. W. et Spooner, S., J. Appl. Phys. 41 (1970) 1319.

[5] Vincze, I., Campbell, I. A. et Meyer, A. J., Solid State Commun 15-9 (1974) 1495.

[6] Chandra, G., Bansal, C. et Ray, J., Phys. Stat. Sol. (a) 35 (1976) 73.

[7] Eymery, J. P., Thèse de Doctorat, Poitiers (1974).

[8] Rhodes, E., Polinger, A., Spijkerman, J. J. et Christ, B. W., Trans. AIME 242 (1968) 1922.

[10] DriJVER, J. W., Thèse de Doctorat, Gröningen (1975).

.[11] Kouvel, J. S., Magnetism and Metallurgy, edited by Berkowitz A. and Kneller E., 1969 Vol. 2 ch. XI.

[12] Collins, M. F. et Forsyth, J. B., Phil. Mag. 8 (1963) 401.

[13] Muraoka, Y., Shiga, M., Yasuoka, H. et Nakamura, Y., J. Phys. Soc. Japan, 402 (1976) 414.

[14] English, A. T., Trans. AIME 236 (1966) 14

[15] Krahl, D., 4th Eur. Reg. Conf. on Electron Microscopy, Rome, edited by Bocciarelli D. S. 11 (1968) 441.

[16] Demenet, J. L., Eymery, J. P. et Moine, P., Rev. Phys. Appl. 9 (1974) 721.

[17] Grosbras, P., Eymery, J. P. et Moine, P., Acta Metall. 24 (1976) 189. 